INPLASY

PROTOCOL

To cite: Yu et al. Anlotinibcontaining regimen for advanced small-cell lung cancer: A protocol of metaanalysis. Inplasy protocol 202110034. doi:

10.37766/inplasy2021.1.0034

Received: 11 January 2021

Published: 12 January 2021

Corresponding author:

Kan Xu

xukanmed@163.com

Author Affiliation:

Affiliated Hangzhou Chest

Hospital, Zhejiang University

School of Medicine

Support: 20201203B179, 2015 PYA008.

Review Stage at time of this submission: The review has not yet started.

Conflicts of interest:

None.

\section{Anlotinib-containing regimen for advanced small-cell lung cancer: A protocol of meta-analysis}

Yu, G1; Cai, Q2; Xu, X3; Shen, Y4; Xu, K5.

Review question / Objective: Small cell lung cancer (SCLC) is a highly malignant lung cancer with a very poor prognosis. To improve the prognosis of SCLC, effective treatment options are needed, but current clinical treatment options are still limited, especially for patients who have failed first or second line therapy. The application of anlotinib as a potentially beneficial new treatment option for SCLC is also very convenient. The aim of this meta-analysis is to evaluate the efficacy and safety of anlotinib-containing regimen for the treatment of SCLC.

Information sources: We will search SinoMed, Wanfang Database, China National Knowledge Infrastructure (CNKI), Embase, Cochrane Library, and PubMed for relevant articles that may meet the criteria published before January 10, 2021. We will also evaluate whether the references cited in the reviews meet the criteria to find additional articles.

INPLASY registration number: This protocol was registered with the International Platform of Registered Systematic Review and Meta-Analysis Protocols (INPLASY) on 12 January 2021 and was last updated on 12 January 2021 (registration number INPLASY202110034).

\section{INTRODUCTION}

Review question / Objective: Small cell lung cancer (SCLC) is a highly malignant lung cancer with a very poor prognosis. To improve the prognosis of SCLC, effective treatment options are needed, but current clinical treatment options are still limited, especially for patients who have failed first or second line therapy. The application of anlotinib as a potentially beneficial new treatment option for SCLC is also very convenient. The aim of this meta-analysis is to evaluate the efficacy and safety of 
anlotinib-containing regimen for the treatment of SCLC.

Condition being studied: Platinumcontaining chemotherapy is the first-line option for SCLC treatment. Although most SCLC responds well to first-line chemotherapy, a majority of SCLC relapses and progresses within 6 months. Some studies have shown that SCLC can benefit from immunotherapy, but its application is still limited by its high price and some of its serious toxic side effects. Current treatment modalities are limited when progression after chemotherapy and/or immunotherapy. These characteristics of SCLC make its 5-year survival rate quite low, only about $2 \%$. Neovascularization plays an important role in the growth, proliferation and metastasis of solid tumors. Anti-angiogenic drugs can inhibit tumor neovascularization, degrade existing tumor blood vessels and reduce tumor blood supply, thus inhibiting tumor growth. Anti-angiogenic therapy is emerging as an effective anti-tumor treatment in addition to chemotherapy, targeted therapy and immunotherapy, which is also applicable to lung cancer. Anlotinib, an oral novel smallmolecule multitarget tyrosine kinase inhibitor (TKI), which could inhibit tumor angiogenesis and proliferation. By inhibiting tumor blood supply, anlotinib has shown promising therapeutic effects in a variety of tumors, such as endometrial, ovarian, and cervical cancers . Anlotinib has also shown very good therapeutic efficacy in NSCLC and benefits in SCLC.

\section{METHODS}

Search strategy: \#1 "Small Cell Lung Carcinoma"[Mesh] OR "Small Cell Lung Cancer" OR "Oat Cell Lung Cancer" OR "Small Cell Cancer of The Lung" OR "Carcinoma, Small Cell Lung" OR "Oat Cell Carcinom a of Lung" \#2 "anlotinib" [Supplementary Concept] OR AL3818 \#3 \#1 AND \#2.

Participant or population: XParticipants with histopathologically or cytologically confirmed advanced SCLC and treated with anlotinib-containing regimen or other regimens.

Intervention: Anlotinib-containing regimen as an intervention in the observation group.

Comparator: Anlotinib-containing regimen as an intervention in the observation group.

Study designs to be included: Clinical randomized controlled trials (RCTs).

Eligibility criteria: Types of studies: Clinical randomized controlled trials (RCTs) comparing anlotinib-containing regimens with other treatment regimens for advanced SCLC will be included in this study. Retrospective studies, single arm studies, articles published in languages other than Chinese or English, conference reports, studies with only abstracts reported but no full texts, and case reports will be excluded. Types of participants Participants with histopathologically or cytologically confirmed advanced SCLC and treated with anlotinib-containing regimen or other regimens. We do not apply any restrictions in terms of age, gender, and ethnicity. Types of interventions: Anlotinib-containing regimen as an intervention in the observation group. c Outcomes: The primary outcomes of this study will be progression-free survival (PFS) and overall survival (OS). The secondary outcomes will be the objective response rate (ORR), disease control rate (DCR), and adverse events (AEs).

Information sources: We will search SinoMed, Wanfang Database, China National Knowledge Infrastructure (CNKI), Embase, Cochrane Library, and PubMed for relevant articles that may meet the criteria published before January 10, 2021. We will also evaluate whether the references cited in the reviews meet the criteria to find additional articles.

Main outcome(s): The primary outcomes of this study will be progression-free survival (PFS) and overall survival (OS).

Additional outcome(s): The secondary outcomes will be the objective response 
rate (ORR), disease control rate (DCR), and adverse events (AEs).

Data management: The candidate articles obtained from the search will be imported into Endnote X9.2 for management. After removing duplicate articles through Endnote, two authors will independently investigate the title, abstract and then full texts of each article to determine whether the article meet the inclusion criteria designed in this protocol. When there are disagreements between the two authors, it will be resolved by discussing with a third author. The same two authors in the study screening phase will independently extract the relevant data from the included articles. They will cross-check to find inconsistent data and discuss with a third author to resolve the disagreement. We will extract the following data from the included articles: country, name of first author, publication year, study design, number of participants, participants characteristics (such as age, sex, smoking history, and brain metastasis), treatment regimen, number of lines of therapy, and median PFS, OS with hazard ratios (HRs) and their 95\% confidence intervals (Cls), ORR, DCR, and AEs.

Quality assessment / Risk of bias analysis: The same two authors will independently assess the risk of bias of each included article. The risk of bias will be evaluated for each included study using the Cochrane Handbook for Systematic Reviews of Interventions (20). We will assess the risk of bias according to the following ranges: selection bias, performance bias, detection bias, attrition bias, reporting bias, and other biases. If more than $\mathbf{1 0}$ articles are included, then we will use funnel plots for publication bias assessment (21). If publication bias exists, we will use the fill and trim method to further analyze publication bias in the studies.

Strategy of data synthesis: We will use RevMan 5.3 software (Copenhagen: The Nordic Cochrane Centre, The Cochrane Collaboration, 2014) for statistical analysis of the data. The Engauge Digitizer 4.1 software will be used to extract survival data from the Kaplan-Meier curves if only Kaplan-Meier curves are shown in the study and median PFS and/or OS with HRs are not directly reported. We will calculate pooled HRs for PFS and OS, risk ratios (RRs) for ORRs and DCRs, and odds ratios (ORs) for different AEs. Q-statistic will be used to evaluate the statistical heterogeneity between studies (22). Heterogeneity between studies will be considered statistically significant when the P-value of the Q-statistic is less than 0.1 or an 12 is greater than $50 \%$ (23). Data will be analyzed using a fixed-effects model when heterogeneity between studies is insignificant and a random-effects model when heterogeneity between studies was significant.

Subgroup analysis: Subgroup analyses will be performed on various parameters, such as age, sex, smoking history, number of lines of therapy, brain metastasis, and number of metastases to reduce heterogeneity.

Sensibility analysis: Subgroup analyses will be performed on various parameters, such as age, sex, smoking history, number of lines of therapy, brain metastasis, and number of metastases to reduce heterogeneity.

\section{Language: No restriction.}

\section{Country(ies) involved: China.}

Other relevant information: We will evaluate all the strength of the body of evidence based on The Grading of Recommendations Assessment, Development and Evaluation (GRADE) guideline. The quality of evidence will be classified into 4 levels: high, moderate, low, and very low.

Keywords: Anlotinib, small cell lung cancer, efficacy, toxicity, meta-analysis.

Contributions of each author:

Author 1 - Guocan Yu - The author drafted the manuscript, searched databases, selected literatures, managed data and assessed quality. 
Email: dabaitwo@163.com

Author 2 - Qingshan Cai - The author searched databases, selected literatures, managed data and evaluated quality.

Email: caiqs66@163.com

Author 3 - Xudong Xu - The author drafted and revised the manuscript.

Email: xuxudong234@163.com

Author 4 - Yanqin Shen - The author provided statistical expertise and read the final manuscript.

Email: yanqinshen@yeah.net

Author 5 - Kan Xu - The author provided statistical expertise, read, feedback and approved the final manuscript.

Email: xukanmed@163.com 